\title{
BMJ Open Cohort profile: Study of Transition, Outcomes and Gender (STRONG) to assess health status of transgender people
}

\author{
Virginia P Quinn, ${ }^{1}$ Rebecca Nash, ${ }^{2}$ Enid Hunkeler, ${ }^{3}$ Richard Contreras, ${ }^{1}$ \\ Lee Cromwell, ${ }^{4}$ Tracy A Becerra-Culqui, ${ }^{1}$ Darios Getahun, ${ }^{1}$ Shawn Giammattei, ${ }^{5}$ \\ Timothy L Lash, ${ }^{2}$ Andrea Millman, ${ }^{6}$ Brandi Robinson, ${ }^{4}$ Douglas Roblin, ${ }^{7}$ \\ Michael J Silverberg, ${ }^{6}$ Jennifer Slovis, ${ }^{8}$ Vin Tangpricha, ${ }^{9,10}$ Dennis Tolsma, ${ }^{4}$ \\ Cadence Valentine, ${ }^{1}$ Kevin Ward, ${ }^{2}$ Savannah Winter, ${ }^{4}$ Michael Goodman ${ }^{2}$
}

To cite: Quinn VP, Nash R, Hunkeler $\mathrm{E}$, et al. Cohort profile: Study of Transition, Outcomes and Gender (STRONG) to assess health status of transgender people. BMJ Open 2017;7:e018121. doi:10.1136/ bmjopen-2017-018121

- Prepublication history and additional material for this paper are available online. To view these files, please visit the journal online (http://dx.doi. org/10.1136/bmjopen-2017018121).

Received 13 June 2017 Revised 11 September 2017 Accepted 13 November 2017

CrossMark

For numbered affiliations see end of article.

Correspondence to Dr Michael Goodman; mgoodm2@emory.edu

\section{ABSTRACT}

Purpose The Study of Transition, Outcomes and Gender (STRONG) was initiated to assess the health status of transgender people in general and following genderaffirming treatments at Kaiser Permanente health plans in Georgia, Northern California and Southern California. The objectives of this communication are to describe methods of cohort ascertainment and data collection and to characterise the study population.

Participants A stepwise methodology involving computerised searches of electronic medical records and free-text validation of eligibility and gender identity was used to identify a cohort of 6456 members with first evidence of transgender status (index date) between 2006 and 2014. The cohort included 3475 (54\%) transfeminine (TF), 2892 (45\%) transmasculine (TM) and $89(1 \%)$ members whose natal sex and gender identity remained undetermined from the records. The cohort was matched to 127608 enrollees with no transgender evidence (63825 women and 63783 men) on year of birth, race/ethnicity, study site and membership year of the index date. Cohort followup extends through the end of 2016.

Findings to date About $58 \%$ of TF and $52 \%$ of TM cohort members received hormonal therapy at Kaiser Permanente. Chest surgery was more common among TM participants ( $12 \%$ vs $0.3 \%)$. The proportions of transgender participants who underwent genital reconstruction surgeries were similar $(4 \%-5 \%)$ in the two transgender groups. Results indicate that there are sufficient numbers of events in the TF and TM cohorts to further examine mental health status, cardiovascular events, diabetes, HIV and most common cancers. Future plans STRONG is well positioned to fill existing knowledge gaps through comparisons of transgender and reference populations and through analyses of health status before and after gender affirmation treatment. Analyses will include incidence of cardiovascular disease, mental health, HIV and diabetes, as well as changes in laboratory-based endpoints (eg, polycythemia and bone density), overall and in relation to gender affirmation therapy.

\section{Strengths and limitations of this study}

- Perhaps the most important strength of this study is systematic cohort identification without a need for participant opt-in.

- Additional strengths inlude accurate determination of gender identity and comprehensive ascertainment of hormonal and surgical treatment received at Kaiser Permanente.

- Notable weaknesses of the study include relatively short follow-up, dearth of information on gender affirmation treatment received outside of the health plans and lack of data on outcomes not captured in the medical records.

\section{INTRODUCTION}

Transgender people are a diverse group of individuals whose biological sex does not match their gender identity. ${ }^{1}$ Typically, sex is assigned at birth based on the appearance of the genitalia. ${ }^{2}$ In contrast, an individual's gender identity is defined as being a male/man, female/woman or of a different gender. ${ }^{23}$ Many transgender people may not self-identify based on binary definitions; ${ }^{4}$ however, a person whose gender identity differs from a male natal sex assignment is often referred to as male-to-female or trans woman, and a person whose gender identity differs from a female natal sex is often referred to as a female-to-male or trans man. ${ }^{56}$ More recently, the terms transfeminine (TF) and transmasculine (TM) have become preferred as they also apply to individuals who do not identify with binary gender categories. ${ }^{7}$

Transgender individuals sometimes seek medical gender affirmation, which may involve administration of cross-sex hormone therapy (HT) to achieve desired masculinisation or 


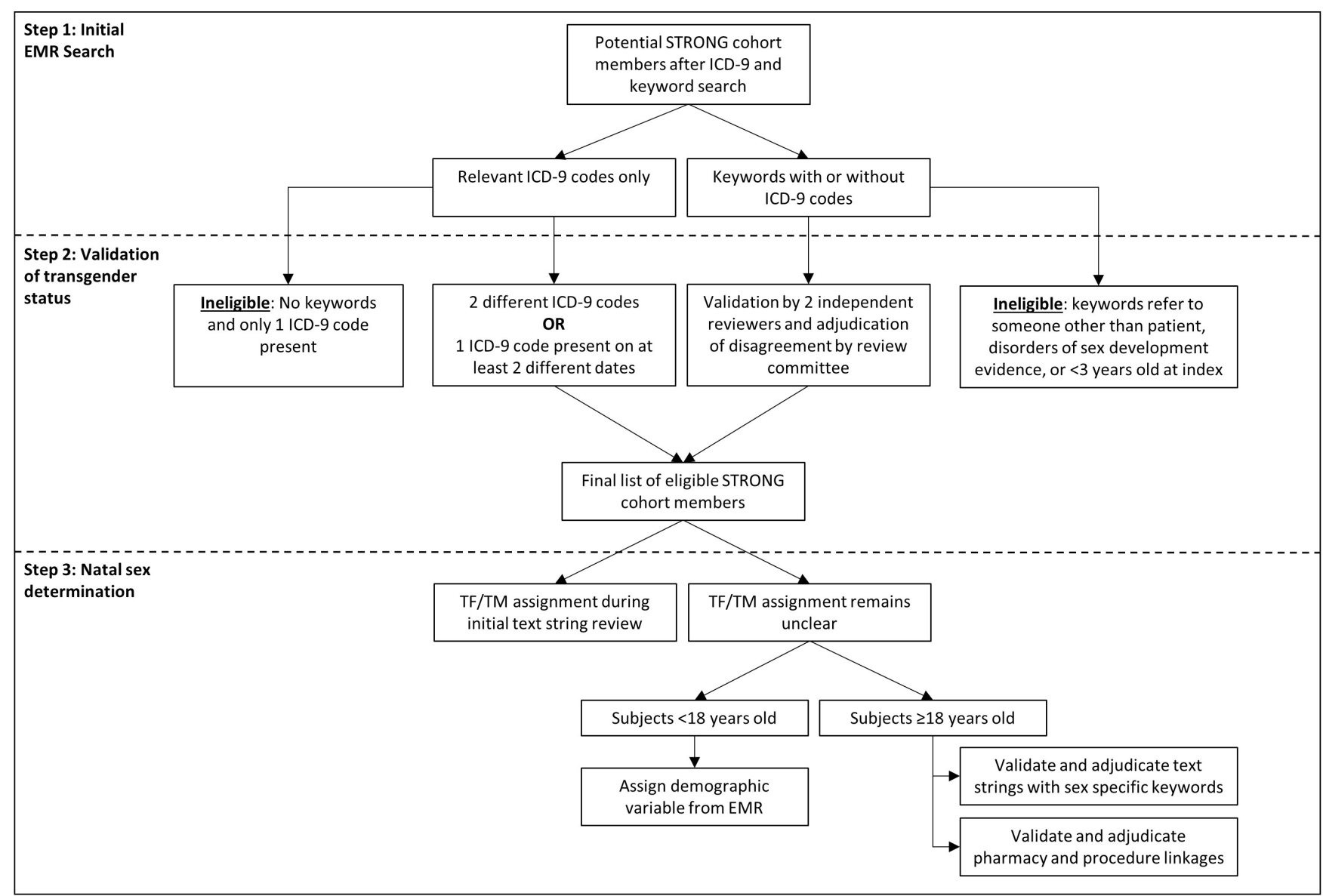

Figure 1 STRONG transgender cohort ascertainment flow diagram. EMR, electronic medical record; ICD-9; International Classification of Diseases, Ninth edition; STRONG, Study of Transition, Outcomes and Gender; TF, transfeminine; TM, transmasculine.

feminisation, and/or surgical change of the genitalia and other sex characteristics. ${ }^{89}$ Although several organisations have established guidelines for clinical care of transgender patients, ${ }^{9}{ }^{10}$ many issues in transgender health and gender affirmation therapy remain unresolved due to lack of direct evidence. ${ }^{11}$ Consequently, many current practices and standards of care are based on expert opinion, case reports or extrapolation of research findings from other populations. For example, current recommendations regarding risk of venous thromboembolism in $\mathrm{TF}$ patients receiving oestrogen are based on the observed effects of hormone replacement therapy in postmenopausal women. ${ }^{12}{ }^{13}$ Similarly, expected health risks in TM are inferred from comorbidities associated with polycystic ovary syndrome. ${ }^{14}$

Critical knowledge gaps include the effect of HT and surgery on gender dysphoria (the feeling of distress when natal sex does not match gender identity ${ }^{15}$ ) and other mental health issues, haematological side effects of HT and risk of cardiovascular disease, metabolic or endocrine disorders and cancer following hormonal or surgical gender affirmation. ${ }^{16}$ A direct evaluation of some of these issues requires longitudinal studies with large numbers of TM and TF participants with sufficient follow-up and variable history of surgical interventions and cross-sex HT use. ${ }^{17}$ These considerations motivated the design of the longitudinal cohort for 'Study of Transition, Outcomes and Gender (STRONG)'. This paper provides a summary of the challenges facing transgender health research, describes the main elements of the STRONG study design and data collection and discusses lessons learnt during the implementation of this project. In this 'cohort profile' communication, we offer a detailed documentation of methods used to assemble, validate and characterise the STRONG cohort and offer an overall description of the study population that will provide data for a multitude of subsequent hypothesis-testing studies.

\section{METHODOLOGICAL CHALLENGES FACING TRANSGENDER HEALTH STUDIES}

The methodological challenges in observational studies of transgender health fall into five categories: (1) attaining sufficient sample size and statistical power; (2) systematic and comprehensive identification of eligible study participants and comparable reference groups; (3) determination of natal sex and/or gender identity; (4) assessment of current and past gender affirmation treatment and (5) engagement of patient and physician stakeholders at all stages of research. ${ }^{17}$ 
Table 1 ICD-9 codes and keywords used to identify potentially eligible STRONG transgender cohort members among KPGA, KPSC and KPNC members

\section{ICD-9 diagnostic codes}

302.5-trans-sexualism

302.50 - trans-sexualism with unspecified sexual history (aka 'trans-sexualism not otherwise specified') 302.51 -trans-sexualism with asexual history 302.52 - trans-sexualism with homosexual history 302.53 - trans-sexualism with heterosexual history 302.85-gender identity disorder in adolescents or adults

302.6- gender identity disorder in children 302.3 - transvestic fetishism

\begin{tabular}{|c|c|}
\hline ICD-9 V codes*+internal KP codes & Keywords \\
\hline $\begin{array}{l}\text { V49.89+121141596-other conditions influencing } \\
\text { health: transgender } \\
\text { V45.77+121141596-acquired absence of genital } \\
\text { organs: history of sex reassignment surgery } \\
\text { V07.8+12124952-other specified prophylactic } \\
\text { measure: male-to-female hormone supplementation } \\
\text { V07.8+12124310-other specified prophylactic } \\
\text { measure: female-to-male hormone supplementation }\end{array}$ & $\begin{array}{l}\text { 'Transgender' } \\
\text { 'Transsexual' } \\
\text { 'Transvestite' } \\
\text { 'Gender } \\
\text { identity' } \\
\text { 'Gender } \\
\text { dysphoria' } \\
\text { 'Gender } \\
\text { reassignment' }\end{array}$ \\
\hline
\end{tabular}

${ }^{*}$ ICD-9 V codes are used for supplementary classification of factors influencing health status. ${ }^{4041}$ As $\mathrm{V}$ codes may cover several conditions, they have to be used in conjunction with internal KP codes to ensure specificity.

ICD-9, International Classification of Diseases Ninth edition; KP, Kaiser Permanente; KPGA, Kaiser Permanente health plan located in Georgia; KPNC, Kaiser Permanente health plan located in Northern California; KPSC, Kaiser Permanente health plan located in Southern California; STRONG, Study of Transition, Outcomes and Gender.

Transgender people represent a hard-to-reach population, and to date most existing cohorts assessing transgender health were assembled in specialised clinics that provide gender affirmation care. ${ }^{18}$ This approach provides good options for collecting detailed treatment data and biospecimens but may exclude individuals who have not sought or who have already completed treatment, and makes it difficult to select comparable reference groups. ${ }^{20}$ In addition, establishing a large clinic-based cohort requires coordination across multiple sites, is costly and may still have small numbers resulting in studies of relatively low statistical power. ${ }^{17}$

Besides clinic-based studies, previous efforts to identify transgender individuals for health research also involved population surveys ${ }^{21}$ and reviews of electronic records for relevant International Classification of Diseases (ICD) codes. $^{22}{ }^{23}$ While surveys offer generalisable population estimates, they may be affected by recall bias and low response rates and require large-scale efforts to identify sufficient numbers of transgender people. Reliance on ICD codes allows identifying large samples from insurance claims or electronic medical records (EMRs), but may exclude eligible study participants who do not have or do not wish to receive a transgender-specific diagnosis. ${ }^{24}$

A critical aspect of transgender research is accurate identification of gender identity. In recent years, the US Department of Health and Human Services issued a directive that EMR systems should enable providers to record gender identity and sexual orientation. ${ }^{25}$ This directive should improve documentation over time; but in the meantime, determination of TF or TM status presents a methodological challenge because the available demographic data can reflect natal sex or gender identity, without specifying which is which. Assessing TM/TF

Table 2 Keywords used for STRONG transgender cohort natal sex assignment

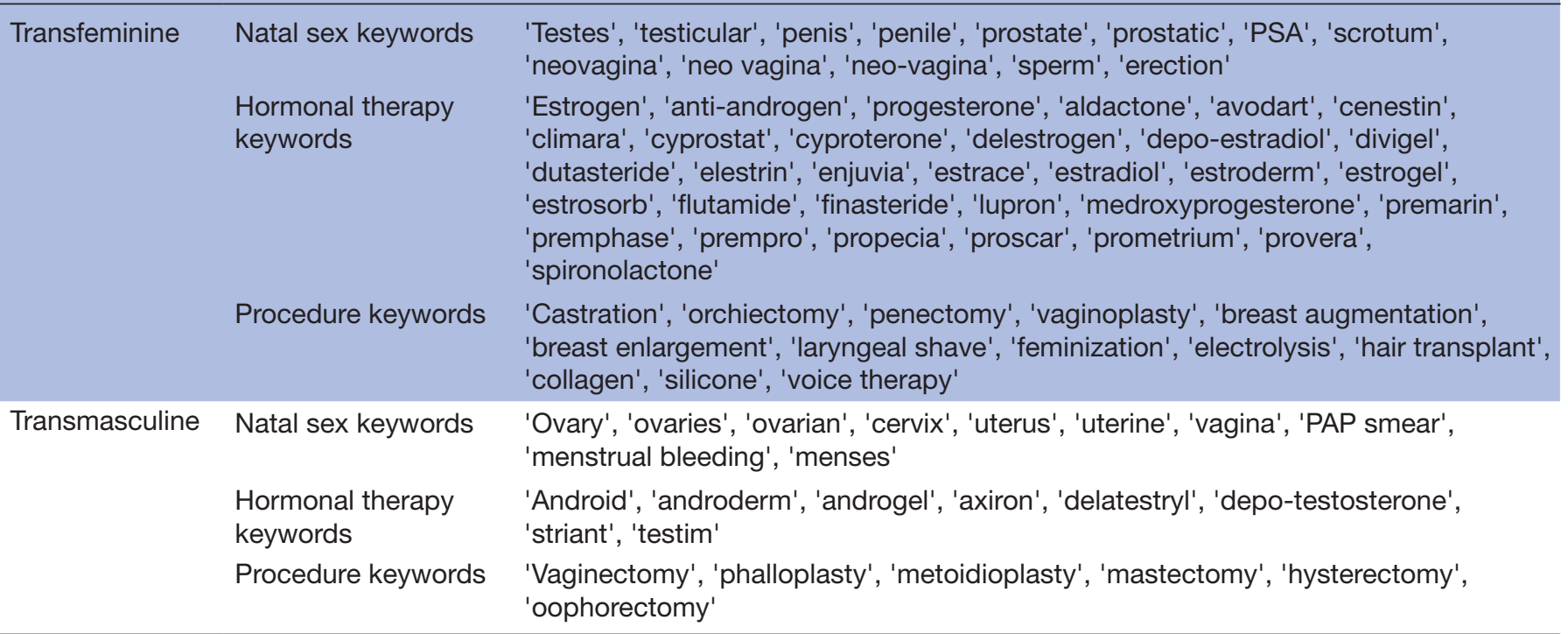

PAP, Papanicolau; PSA, prostate specific antigen; STRONG, Study of Transition, Outcomes and Gender. 
Box Data available for Study of Transition, Outcomes and Gender transgender cohort

\author{
Data categories and specific elements \\ Demographic and membership characteristics \\ - Age, sex and race/ethnicity \\ - Health plan site \\ - Area-based socioeconomic status factors* \\ - Enrolment/disenrolment intervals \\ - Insurance plan type \\ General health indicators \\ - Height/weight (body mass index)* \\ - Smoking status* \\ - Comorbidities \\ Gender affirmation procedures \\ - Current Procedure Terminology and/or International Classification of \\ Diseases code \\ Date of procedure \\ - History of gender affirmation procedures (clinical notes) \\ Pharmacy records (hormone therapy, psychiatric medications) \\ - Medication prescribed \\ Filled prescription for medication \\ $\rightarrow$ Dose \\ - Form \\ Dates of prescription and fill \\ Visit-associated diagnoses \\ - Cardiovascular disease \\ Diabetes \\ $>$ HIV \\ Mental health problems \\ Cancer diagnoses \\ $\rightarrow$ Stage \\ > Site \\ - Histology \\ Date of diagnosis \\ Laboratory results \\ $\rightarrow$ Laboratory test \\ - Value \\ - Date \\ Vital status \\ $\rightarrow$ Date of death \\ - Cause of death
}

Note: *Assessed at index date (date of first evidence of transgender status in electronic medical record).

status can be achieved by asking two questions about natal sex and gender identity ${ }^{26}$; however, reliance on self-report requires contact with individual participants and is subject to non-response, which increases the risk of selection bias.

Insurance coverage for gender affirmation therapy has increased over time ${ }^{27-29}$; however, it remains sporadic and incomplete, and many transgender people seek care outside of regular healthcare plans. ${ }^{30}$ This presents a specific challenge for any study that aims to ascertain hormonal exposures and surgical procedures, particularly for transgender people with no or inadequate insurance coverage and for persons who initiated gender-affirming therapy years ago.
In a rapidly developing field, such as transgender health, even experienced researchers may lack specific expertise required to prioritise research questions and select the most relevant patient-centred outcome measures. Thus, it is important to involve members of the transgender community and their physicians to ensure that the proposed research questions, study design and data collection methods are relevant, appropriate and feasible. ${ }^{31}$ Conversely, as the research methods and interpretation of findings are becoming increasingly complex, researchers should communicate with stakeholders directly to convey information relevant to their decision-making. ${ }^{32}$

In sum, transgender health research faces significant methodological challenges and logistical barriers. These challenges and barriers contribute to the lack of knowledge about the health risks in this population and preclude development of evidence-based recommendations for transgender healthcare. ${ }^{11}$

\section{COHORT DESCRIPTION}

Study goals, design and setting

STRONG was initiated in September 2013 with the primary long-term goal of assessing the health status of transgender individuals overall, among TF/TM subgroups, and in both subgroups after different types of gender-affirming treatment. It was designed as an EMR-based retrospective/prospective cohort study of transgender members enrolled in three Kaiser Permanente (KP) health plans located in Georgia (KPGA), Northern California (KPNC) and Southern California (KPSC). These health plans are prepaid integrated care systems and currently provide comprehensive health services to approximately 8 million members. Individuals and their families may enrol through an employer, state or federal programmes such as Medicaid and Medicare, or directly. The populations of enrollees are sociodemographically diverse and broadly representative of the communities in the corresponding areas. ${ }^{33} 34$

The three KP organisations are members of several research consortia including the Healthcare Systems Research Network ${ }^{35}$ and the Mental Health Research Network. ${ }^{36}$ They share similarly structured databases organised into 'Virtual Data Warehouses' (VDW), with files stored behind security firewalls at each site. The files have identical variable names, formats and specifications that allow using centrally generated and/or distributed programs to create harmonised analytic datasets. ${ }^{37}$ VDW files are linked by unique individual identifiers, allowing researchers to construct historical and prospective cohorts. ${ }^{38}$

The study was conducted in partnership with the Emory University, which served as the coordinating centre. All activities described in this manuscript were reviewed and approved by the Institutional Review Boards (IRBs) of the four institutions. 


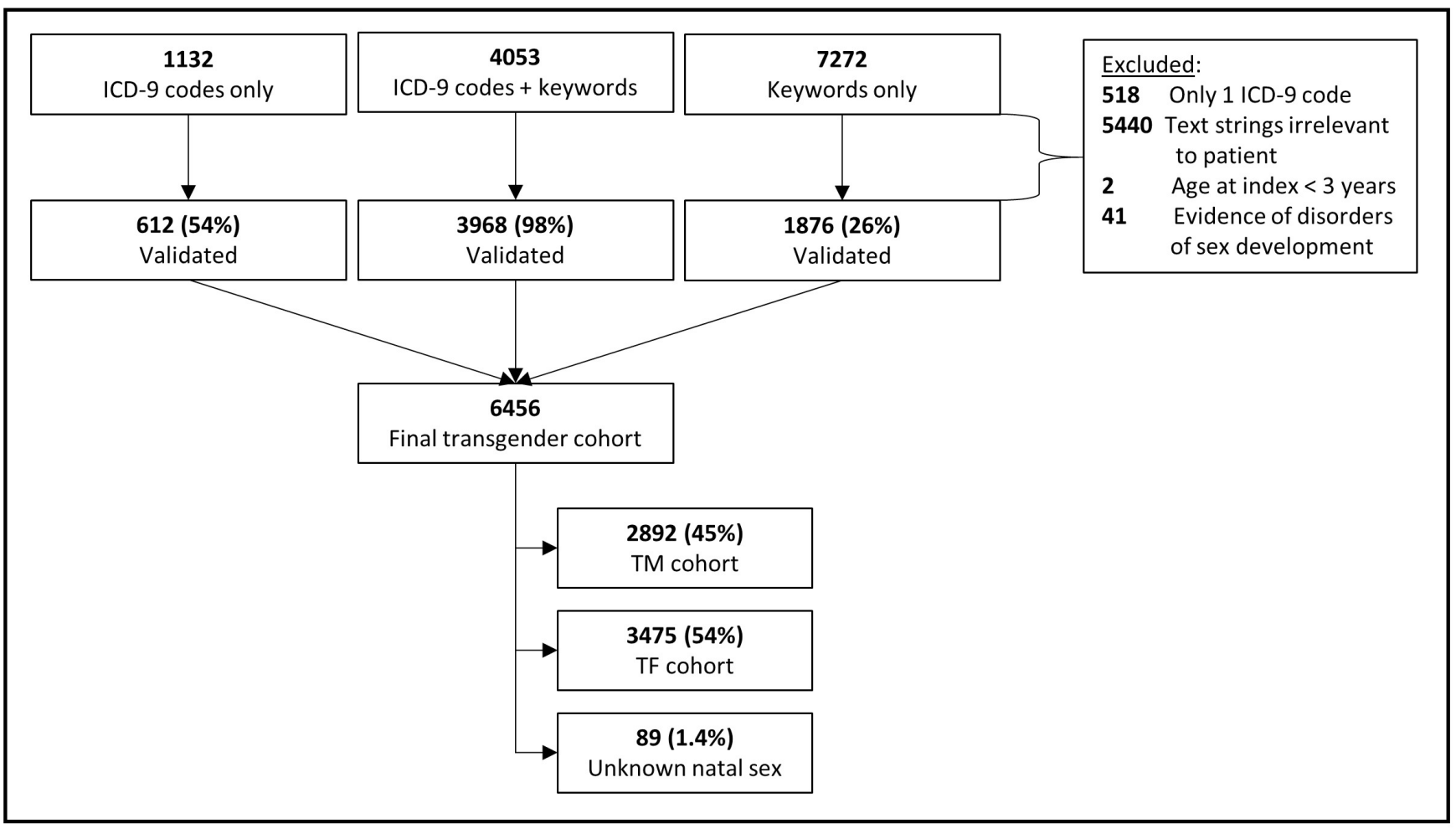

Figure 2 Results of STRONG transgender cohort ascertainment and validation. ICD-9; International Classification of Diseases Ninth edition; STRONG, Study of Transition, Outcomes and Gender; TF, transfeminine; TM, transmasculine.

The stakeholder involvement was achieved by assembling a project advisory group. To recruit the Stakeholder Advisory Group members, investigators at each site were charged with identifying a leading clinician specialising in transgender care. The physicians were then asked to nominate one or two patients to serve as representatives of the transgender community. The resulting Stakeholder Advisory Group included 12 members. The stakeholders made a number of important contributions to the project at various stages of study design, planning and implementation, as described below.

\section{Cohort ascertainment}

Figure 1 shows the three-step algorithm used to identify transgender cohort members. It includes initial EMR search to identify cohort candidates (step 1), validation of transgender status (step 2) and determination of TM/ TF status (step 3).

\section{Step 1: Initial EMR search}

A computer programme written using Statistical Analysis Software (SAS) V.9.4 (SAS Institute Inc., Cary, NC) was used to search the EMRs of KPGA, KPNC and KPSC members of all ages enrolled between 1 January 2006 and 31 December 2014 to identify two types of evidence supporting transgender status: (1) relevant International Classification of Diseases, Ninth edition (ICD-9) codes and (2) presence of relevant specific keywords in freetext clinical notes (table 1). The program was developed and pilot-tested at KPGA and then distributed to the remaining sites. Cohort ascertainment was undertaken before the health plans switched to ICD-10 codes.

The diagnostic ICD-9 codes suggestive of transgender status were selected based on consultations with the STRONG Stakeholder Advisory Group and methodologies described in earlier studies. ${ }^{23} 39$ Transvestic fetishism (ICD-9 code 302.3) was included based on previous observations that men who initially meet criteria for this diagnostic category may later experience persistent gender dysphoria consistent with transgender status. ${ }^{22}$

We also used ICD-9 V codes, which allow for supplementary classification of factors influencing health status. ${ }^{40} 41$ As $\mathrm{V}$ codes may cover several conditions, we used them in conjunction with internal KP codes to ensure specificity. For example, a combination of ICD-9 code V49.89 and KP code 121141596 means 'Other conditions influencing health: transgender'.

The second method of transgender ascertainment involved another custom-written program that identified the relevant keywords in free-text clinical notes recognising that both appropriate and inappropriate terms could be found in the EMR. During pilot testing, an expanded list of keywords provided by the stakeholders was used; that list was gradually shortened after stepwise removal of keywords that did not contribute additional cases. The resulting list provided a complete cohort ascertainment with the shortest program running time. 


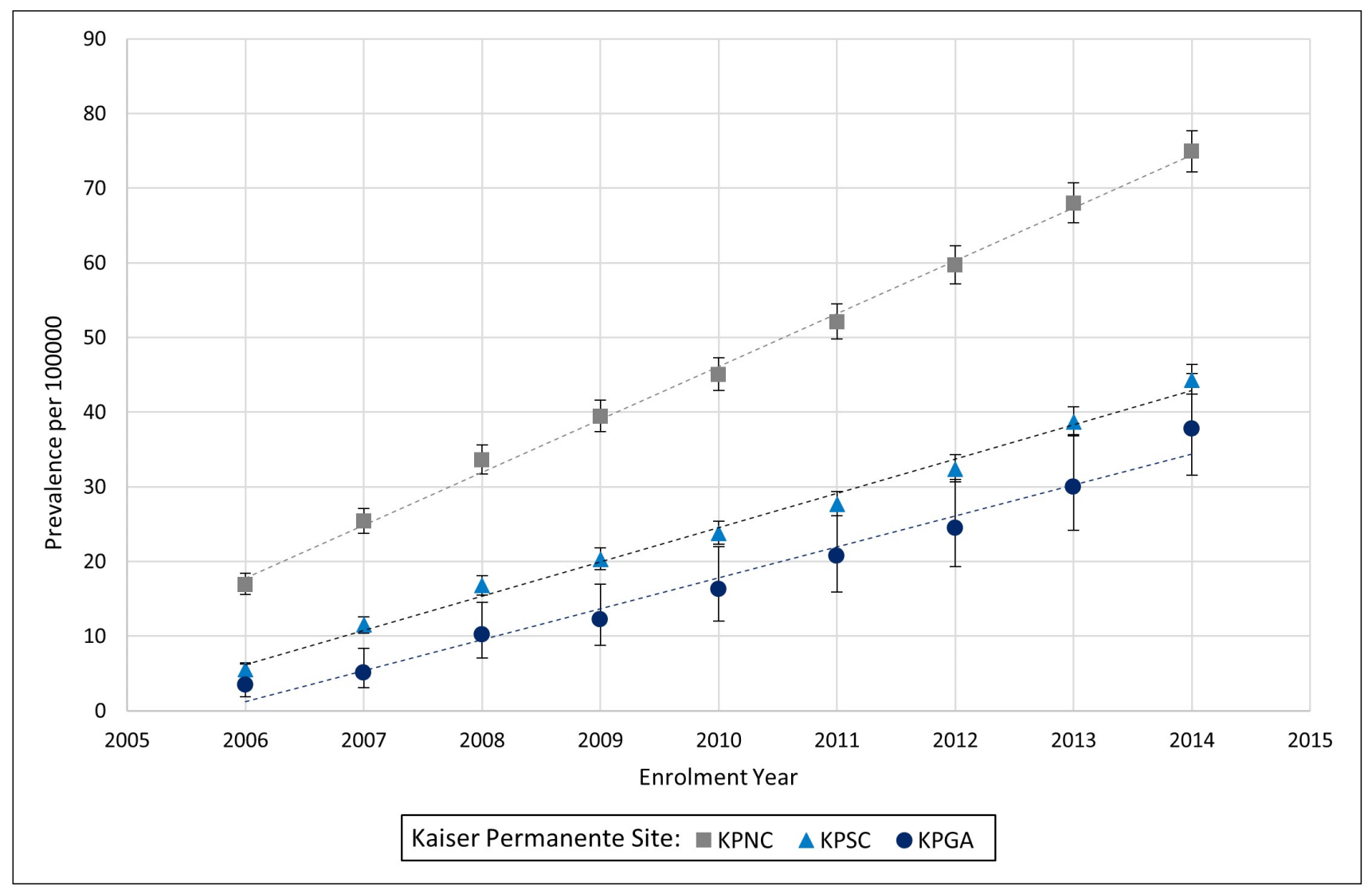

Figure 3 Prevalence of transgender status by site and year of health plan enrolment. Dotted lines represent linear trends. KPGA, Kaiser Permanente health plan located in Georgia; KPNC, Kaiser Permanente health plan located in Northern California; KPSC, Kaiser Permanente health plan located in Southern California.

\section{Step 2: Cohort validation}

A separate program extracted short strings of text that included 100 characters before and 50 characters after each keyword of interest. When clinical notes contained relevant keywords (with or without an ICD-9 code), transgender status was confirmed through an examination of the deidentified text strings by two trained reviewers. Disagreements among reviewers were adjudicated by a review committee that included two physician investigators (MG and VT) and the project manager (RN).

Cohort candidates with no keywords but at least two different diagnostic codes or the same code on different dates were considered eligible. The validity of this approach was confirmed using unstructured chart review during pilot testing of the study protocol, as described previously. $^{24}$

Members who had evidence of disorders of sex development (ie, abnormalities of chromosomal, gonadal or anatomic $\operatorname{sex}^{42}$ ) and those younger than 3 years of age at the index date were excluded.

\section{Step 3: Determination of TF/TM status}

Each eligible study participant was categorised as TF or TM using several methods. We used all keyword text strings and ICD-9 codes extracted for step 1 to identify additional words such as 'male-to-female', 'female-to-male' and gender affirmation V codes (V07.8+12124952 and V07.8+12124310). During the validation of transgender status, the reviewers were also instructed to categorise each eligible person as 'natal male', 'natal female' or 'unclear'.

For persons whose $\mathrm{TF} / \mathrm{TM}$ status was unclear after the initial review and for persons with ICD-9 codes only, another free-text program was developed to search for keywords reflecting natal sex anatomy (eg, 'testes' or 'ovaries'), history of specific procedures (eg, orchiectomy or hysterectomy) or evidence of hormonal therapy (eg, oestrogen or testosterone). The keywords used for assigning $\mathrm{TM} / \mathrm{TF}$ status are included in table 2. Text strings containing TF-specific and TM-specific keywords were reviewed and adjudicated as discussed above.

\section{Gender affirmation treatment status}

During the initial STRONG cohort validation (step 2) and natal sex determination (step 3), reviewers were instructed to check a box for 'Evidence of treatment' if the text strings provided an indication of receipt or referral for HT, surgery or other relevant procedures (eg, electrolysis). Disagreements were adjudicated as described previously. 
Table 3 Characteristics of the STRONG transgender cohort

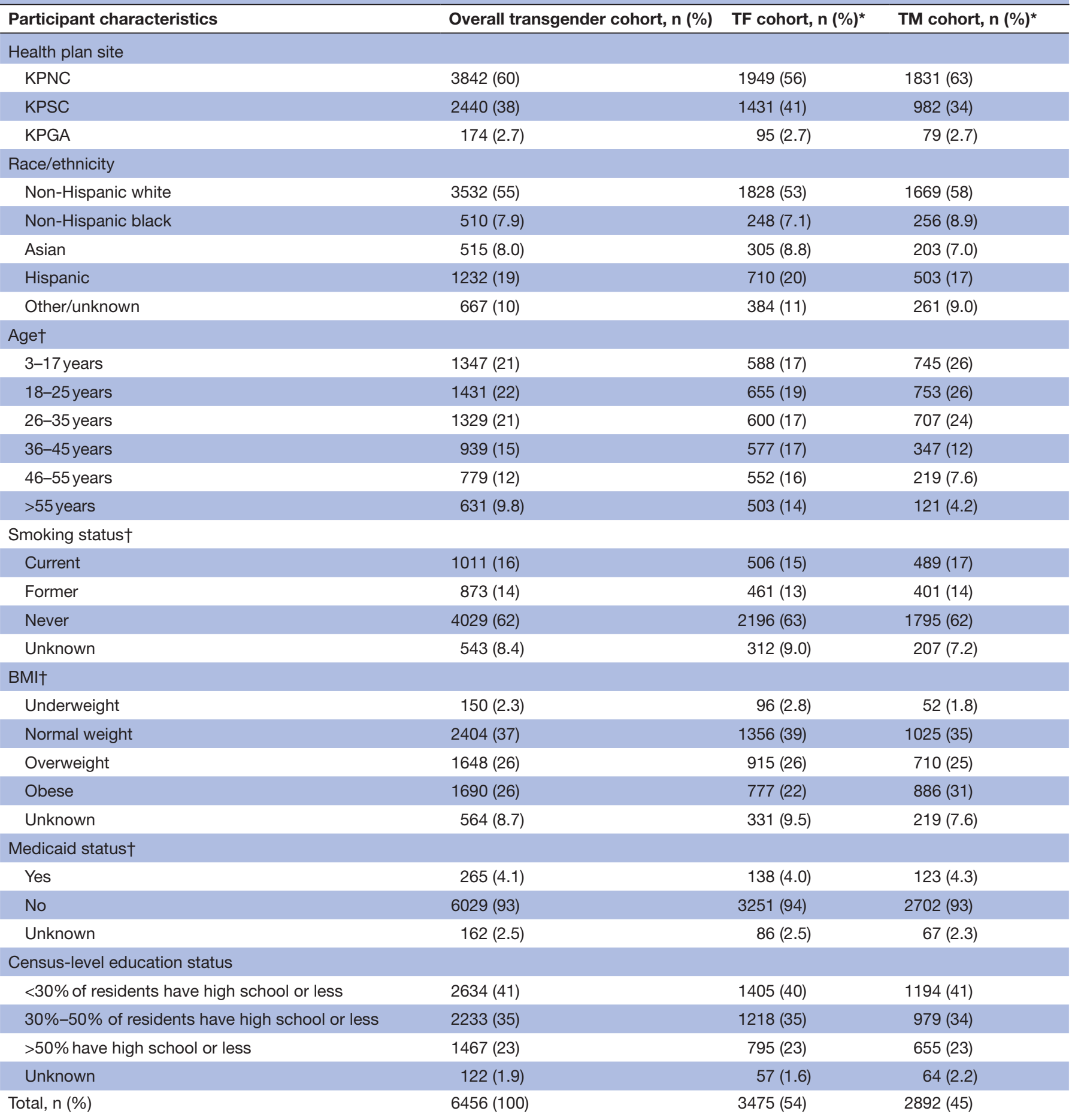

${ }^{*}$ Excludes 89 persons with unknown natal sex.

†Assessed at index date (date of first evidence of transgender status in electronic medical records).

KPGA, Kaiser Permanente health plan located in Georgia; KPNC, Kaiser Permanente health plan located in Northern California; KPSC,

Kaiser Permanente health plan located in Southern California; STRONG, Study of Transition, Outcomes and Gender; TF, transfeminine; TM, transmasculine.

In addition to text string reviews, gender affirmation treatment status was determined by linkage with cross-sex hormone prescriptions using national drug codes, as well as ICD-9, ICD-10 and Current Procedure Terminology (CPT) codes reflecting surgeries and other interventions (online supplementary tables 1-6). TF drugs (eg, oestradiol, spironolactone) in a natal male and TM drugs (eg, testosterone) in a natal female were considered as evidence of HT.

As a result of these steps, cohort members were categorised based on evidence of HT and surgical or other gender affirmation procedures. Gender affirmation 
Table 4 Gender affirmation status of the STRONG transgender cohort members

\begin{tabular}{|c|c|c|c|}
\hline Gender affirmation evidence and type & Overall transgender cohort, n (\%) & TF cohort, n (\%)* & TM cohort, $n(\%)^{*}$ \\
\hline \multicolumn{4}{|l|}{ Evidence of treatment } \\
\hline Any† & $4040(63)$ & $2244(65)$ & $1792(62)$ \\
\hline \multicolumn{4}{|l|}{ Cross-sex hormone therapy } \\
\hline Specific evidence at KP & $3544(55)$ & $2030(58)$ & $1514(52)$ \\
\hline No evidence & $2725(42)$ & $1333(38)$ & $1306(45)$ \\
\hline \multicolumn{4}{|l|}{ Gender affirmation surgery } \\
\hline Top surgery at KP only & $369(5.7)$ & $12(0.3)$ & $357(12)$ \\
\hline Orchiectomy at KP with or without top & $52(0.8)$ & $52(1.5)$ & $0(0)$ \\
\hline $\begin{array}{l}\text { Hysterectomy and/or oophorectomy at KP } \\
\text { with or without top }\end{array}$ & $311(4.8)$ & $0(0)$ & $311(11)$ \\
\hline Evidence in the text only & $432(6.7)$ & $253(7.3)$ & $179(6.2)$ \\
\hline No evidence & $4993(77)$ & $2977(86)$ & $1927(67)$ \\
\hline \multicolumn{4}{|l|}{ Procedures to alter secondary sex characteristics } \\
\hline Specific evidence at KP & $678(11)$ & $385(11)$ & $293(10)$ \\
\hline Evidence in the text only & $58(0.9)$ & $52(1.5)$ & $6(0.2)$ \\
\hline No evidence & $5720(89)$ & $3038(87)$ & $2593(90)$ \\
\hline Total & $6456(100)$ & $3475(100)$ & $2892(100)$ \\
\hline
\end{tabular}

${ }^{*}$ Excludes 89 persons with unknown natal sex.

†Includes non-specific history and referral for treatment.

KP, Kaiser Permanente; STRONG, Study of Transition, Outcomes and Gender; TF, transfeminine; TM, transmasculine.

procedures were categorised as: bottom (eg, vaginoplasty for TF or vaginectomy for TM); top (eg, breast augmentation for TF or mastectomy for TM); interventions to change secondary sex characteristics (eg, electrolysis) or not specified (ie, evidence of surgery in the text only).

\section{Selection of the reference cohort}

$\mathrm{Up}$ to 10 male and 10 female KP enrollees without evidence of transgender status were matched to each member of the final validated transgender cohort on year of birth (within 5-year groups for adults and 2-year groups for children and adolescents), race/ethnicity, KP site and membership year at the index date. Index date was defined as the date of the first recorded evidence of transgender status in the EMR. For some transgender cohort members, the number of reference males or females was less than 10 due to duplicate matches; however, no transgender participant had less than seven referents of either sex. A cluster ID for each matched group was assigned to allow stratified analyses (eg, by HT type or gender-affirming surgery).

\section{Data integration and follow-up}

Patient identification numbers for both the transgender and reference cohorts were linked to multiple data sources to obtain ICD-9 and ICD-10 diagnostic codes for the conditions of primary interest (eg, mental health conditions, cardiovascular diseases and diabetes); disease registries to ascertain incident cancers and HIV diagnoses; psychiatric and behavioural healthcare utilisation; pharmacy records to track mental health treatment and HT receipt over time; and dates and results of laboratory tests including bone scans, blood chemistry analyses, hormone levels and blood cell counts (box). Mortality was ascertained from linkages to death registries. All members of the cohort were assigned a study ID by the programmer at each KP site and no personally identifiable information was included in the aggregated analytic file. To date, cohort follow-up extends through the end of 2016.

\section{FINDINGS TO DATE}

Initial review of the EMRs identified 12457 potential transgender individuals. Of these, 7272 (58\%) were identified through keywords only, $1132(9 \%)$ through ICD-9 codes only and the remaining 4053 (33\%) had both ICD-9 codes and keywords (figure 2). Among these candidates, 6456 were confirmed as transgender: 10\% from ICD-9 codes alone, $29 \%$ from keywords alone and $61 \%$ from both codes and keywords. Based on validation results, the positive predictive values for keywords, diagnostic codes and both were $26 \%, 54 \%$ and $98 \%$, respectively. The leading reason for non-eligibility was the use of a keyword 
Table 5 Frequency of health outcomes in the STRONG TF cohort relative to matched comparison groups

\begin{tabular}{|c|c|c|c|}
\hline Health outcomes & TF cohort, n (\%)* & Reference males, n (\%)* & Reference females, n (\%)* \\
\hline \multicolumn{4}{|l|}{ Cardiovascular disease } \\
\hline Venous thromboembolism & $86(2.5)$ & $670(1.9)$ & $677(2.0)$ \\
\hline Myocardial infarction & $61(1.8)$ & $664(1.9)$ & $319(0.9)$ \\
\hline Peripheral artery disease & $106(3.1)$ & $879(2.6)$ & $645(1.9)$ \\
\hline \multicolumn{4}{|l|}{ Mental health $†$} \\
\hline Anxiety & $1337(38)$ & $4323(13)$ & $7485(22)$ \\
\hline Depression & $1705(49)$ & $4721(14)$ & $8726(25)$ \\
\hline Self-inflicted injury $\ddagger$ & $75(2.2)$ & $100(0.3)$ & $204(0.6)$ \\
\hline Suicidal ideation & $175(5.0)$ & $157(0.5)$ & $194(0.6)$ \\
\hline Colorectum & $10(0.3)$ & $107(0.3)$ & $91(0.3)$ \\
\hline Lung and bronchus & $9(0.3)$ & $58(0.2)$ & $59(0.2)$ \\
\hline Lymphatic and haematopoietic & $22(0.6)$ & $168(0.5)$ & $106(0.3)$ \\
\hline Melanoma & $10(0.3)$ & $227(0.7)$ & $180(0.5)$ \\
\hline Prostate & $25(0.7)$ & $451(1.3)$ & $0(0.0)$ \\
\hline \multicolumn{4}{|l|}{ Other chronic conditions $†$} \\
\hline HIV§ & $186(5.4)$ & $190(0.6)$ & $15(0.0)$ \\
\hline Diabetes & $313(9.0)$ & $3358(9.8)$ & 2619 (7.6) \\
\hline \multicolumn{4}{|l|}{ Vital status } \\
\hline
\end{tabular}

*Percentages do not add to $100 \%$ due to overlapping categories.

†Based on at least two diagnoses in the EMR.

$\ddagger$ Combined diagnoses of self-inflicted injury, self-inflicted injury/poisoning and possible self-inflicted injury.

§HIV status ascertained from disease registries in Kaiser Permanente health plans located in Northern and Southern California and from two or more International Classification of Diseases codes in the EMR in Kaiser Permanente health plan located in Georgia.

EMR, electronic medical record; STRONG, Study of Transition, Outcomes and Gender; TF, transfeminine.

(eg, transgender) referring not to the patient, but to the patient's relative or partner. In other situations, the keywords of interest were used as part of standard text, such as when listing indications for hormone use. Natal sex and/or gender identity was successfully determined for all but $89(1.4 \%)$ of the transgender cohort members.

The transgender cohort was matched to 127608 enrollees with no evidence of transgender status. Of those 63825 were women and 63783 were men.

Figure 3 displays proportions of transgender enrollees over time at each of the three participating sites. In 2006, the prevalence estimates (95\% CIs) per 100000 enrollees were 3.5 (1.9 to 6.3), 5.5 (4.8 to 6.4) and 17 (16 to 19) in KPGA, KPSC and KPNC, respectively. By 2014, the corresponding estimates increased to 38 (32 to 45) in KPGA, 44 (42 to 46) in KPSC and 75 (72 to 78) in KPNC. The composition of the transgender population has also changed. Whereas in 2006, the TF:TM ratio among newly identified cohort members was approximately $1.7: 1$, in 2014 the same ratio was 1:1.

As shown in table 3 , about $60 \%$ of all participants were from KPNC, 38\% were from KPSC and less than 3\% were from KPGA. With respect to race and ethnicity, blacks and Asians each comprised about $8 \%$ of the study population, 19\% were Hispanics and 55\% were non-Hispanic whites. Compared with TF, TM subjects were younger $(76 \%$ vs $53 \%$ under the age of 36) and included a greater proportion of subjects who were obese ( $31 \%$ vs $22 \%)$. Proportions of smokers, insurance status and area-based measures of education were similar in TM and TF study subjects.

Nearly two-thirds of the transgender cohort had some evidence of gender-affirming treatment (table 4). Approximately $55 \%$ of all transgender cohort members had evidence of HT received at KP. This proportion was slightly higher $(58 \%)$ in $\mathrm{TF}$ than in $\mathrm{TM}$ participants (52\%). 
Table 6 Frequency of health outcomes in the STRONG TM cohort relative to comparison groups

\begin{tabular}{|c|c|c|c|}
\hline Health outcomes & TM cohort, n (\%)* & Reference males, n (\%) & Reference females, $\mathbf{n}(\%)^{*}$ \\
\hline \multicolumn{4}{|l|}{ Cardiovascular disease } \\
\hline Venous thromboembolism & $45(1.6)$ & $266(0.9)$ & $356(1.2)$ \\
\hline Stroke & $42(1.5)$ & $360(1.3)$ & $250(0.9)$ \\
\hline Myocardial infarction & $17(0.6)$ & $210(0.7)$ & $88(0.3)$ \\
\hline Peripheral artery disease & $38(1.3)$ & 309 (1.1) & $242(0.8)$ \\
\hline Unstable angina & $20(0.7)$ & $215(0.8)$ & $123(0.4)$ \\
\hline \multicolumn{4}{|l|}{ Mental health $†$} \\
\hline Anxiety & $1323(46)$ & $3583(13)$ & $6089(21)$ \\
\hline Depression & $1594(55)$ & 3806 (13) & $6813(24)$ \\
\hline Self-inflicted injury $\ddagger$ & $121(4.2)$ & $109(0.4)$ & $181(0.6)$ \\
\hline Suicidal ideation & $193(6.7)$ & $160(0.6)$ & $186(0.7)$ \\
\hline Substance abuse disorder & $418(14)$ & $2391(8.4)$ & $1523(5.3)$ \\
\hline \multicolumn{4}{|l|}{ Cancer primary site } \\
\hline Breast & $20(0.7)$ & ी & $217(0.8)$ \\
\hline Cervix & $7(0.2)$ & $0(0.0)$ & $172(0.6)$ \\
\hline Melanoma & $8(0.3)$ & $109(0.4)$ & $71(0.2)$ \\
\hline \multicolumn{4}{|l|}{ Other chronic conditions $†$} \\
\hline HIV§ & $9(0.3)$ & $117(0.4)$ & $10(0.0)$ \\
\hline Diabetes & $153(5.3)$ & $1329(4.7)$ & $1213(4.2)$ \\
\hline \multicolumn{4}{|l|}{ Vital status } \\
\hline Alive & 2853 (99) & 28237 (99) & $28366(99)$ \\
\hline Dead & $39(1.3)$ & $283(1.0)$ & $183(0.6)$ \\
\hline Total & $2892(100)$ & $28520(100)$ & $28549(100)$ \\
\hline
\end{tabular}

${ }^{*}$ Percentages do not add to $100 \%$ due to overlapping categories.

†Based on at least two diagnoses in the EMR.

$\ddagger$ Combined diagnoses of self-inflicted injury, self-inflicted injury/poisoning and possible self-inflicted injury.

§HIV status ascertained from disease registries in Kaiser Permanente health plans located in Northern and SouthernCalifornia and from two or more International Classification of Diseases codes in the EMR in Kaiser Permanente health plan located in Georgia.

ๆCounts $<5$ are suppressed.

EMR, electronic medical record; STRONG, Study of Transition, Outcomes and Gender; TM, transmasculine.

About 23\% of the transgender cohort had some evidence of gender affirmation surgery. Top surgery receipt at KP was far more common among TM cohort members than among their TF counterparts $(12 \%$ vs $0.3 \%)$. Similar proportions of TM and TF cohorts had genital surgeries $(4 \%-5 \%)$ or procedures aimed at altering other secondary sex characteristics $(11 \%)$.

Tables 5 and 6 present the number of cases for various health outcomes in the STRONG population through the end of 2016. These frequencies should not be interpreted as evidence of increased or decreased risk because they do not account for person-time of follow-up, time ordering of the conditions and transgender status and do not take into consideration exposures to cross-sex hormones or surgical procedures. Nevertheless, these data indicate that both TF and TM cohort members, as well as their corresponding referents, have sufficient numbers of cardiovascular events, mental health conditions, HIV, diabetes and several common cancers to permit meaningful analyses. Additional analyses will include changes in laboratory-based endpoints including polycythemia and bone density. These analyses are beyond the scope of this communication, which is focused on the study methods rather than specific findings. The planned analyses will include comparisons of transgender and reference cohorts as well as within-transgender cohort examination of health status before and after surgery and initiation of HT.

\section{STRENGTHS AND LIMITATIONS}

In this communication, we describe STRONG, a health system-based observational study that was designed to examine the health status of transgender people and to evaluate the possible risks and health benefits of various gender-affirming treatments. STRONG aimed to overcome five previously described methodological challenges facing transgender health research.

\section{Sample size and power considerations}

Adequate sample size can be feasibly achieved with the use of large well-defined populations that offer an 
adequate sampling frame. ${ }^{38}$ In practical terms, at least in the USA, this can be done by basing the study in large integrated health systems with millions of members and comprehensive EMRs. The EMR data from the health systems allow assembling cohorts of hard-to-reach populations and ample options for selection of referent groups. The STRONG cohort, which included almost 6500 transgender people and nearly 130000 referents, represents one of the largest studies of its kind available to date. Nevertheless, a number of important analyses by different subtypes of gender affirmation treatment may not be feasible due to sparse stratum-specific data.

\section{Systematic identification of eligible study participants}

We demonstrated that by using a relatively simple algorithm-based on standard codes and supplemented with analysis of digitised provider notes-it is possible to comprehensively identify transgender enrollees of large community-based health plans. The use of keyword-containing text strings enhanced cohort ascertainment relative to ICD code-alone-based approaches. On the other hand, reliance on keywords without text validation would have erroneously included a substantial number of persons who are not transgender. A review of records to confirm transgender status added considerable time and resources; however, it is still more efficient and more comprehensive than the traditional unstructured chart review. In conducting cohort validation, we reviewed up to three clinical note excerpts on 11325 people. This task was accomplished within 6 months.

A comprehensive identification of all transgender people in the KP population (with and without evidence in the medical records) would require contacting more than 8 million members to inquire about their natal sex and gender identity; this does not appear feasible at this time.

\section{Determination of natal sex and/or gender identity}

Our study definitively ascertained natal sex and/or gender identity for nearly $99 \%$ of cohort members. We obtained information on TM/TF status from three sources: keyword text strings, pharmacy records and procedure codes. In most instances, these sources were in agreement; however, in some cases the results were discordant. Each disagreement was used as an opportunity to check data accuracy and allowed reducing misclassification, which would have been substantial if STRONG relied on demographic data in the EMR. For example, among adult TF study subjects, $41 \%$ were documented as 'female' and 59\% were documented as 'male'. The corresponding proportions of people classified as 'female' and 'male' among adult TM cohort members were $59 \%$ and $40 \%$, respectively, with $1 \%$ recorded as unknown in the EMR. By contrast, in $96 \%$ of persons under the age of 18 , the demographic variable reflected natal sex.

A limitation of the current data is the inability to accurately identify persons who reject binary gender categories. These individuals are likely to be found among cohort members who do not have a transgender-specific diagnosis and receive no HT or surgical treatment; however, at the present time EMRs alone are not sufficient for determination of non-binary gender identity. This will be possible in the near future after KP introduces new data capture systems with separate fields for natal sex, gender identity, preferred pronouns, organ inventory and history of gender-affirming procedures.

Assessment of current and past gender affirmation treatment Although the information on gender affirmation received within the KP system is high quality, one of the main limitations of STRONG data is the lack of information on HT and surgical treatment received outside the KP system. This restricts our ability to identify a subcategory of transgender cohort members with no history of gender affirmation treatment of any kind. For this reason, the most definitive analyses are limited to people who initiated therapy at KP. These individuals can be identified among those STRONG participants whose EMR demonstrates a gap between index date and the first prescription for HT. This 'HT initiation' group represents about 35\% of TM and $32 \%$ of TF subjects.

The broadening of coverage for gender affirmation services at KP occurred relatively recently. As the proportion of transgender people among enrollees has been increasing and many patients now initiate and receive gender affirmation therapy exclusively within the system, it is important to both expand the cohort and extend the follow-up of current participants.

\section{Engagement of patient and physician stakeholders}

A critical feature of STRONG is patient-centredness. During the study implementation, we held monthly stakeholder calls and had three inperson Stakeholder Advisory Group meetings. These interactions had direct impact on study design and implementation. For example, the list of keywords used for STRONG cohort ascertainment was proposed, pilot-tested and refined in close consultation with the study stakeholders. Following advice from stakeholders, we expanded eligibility criteria to include transgender and gender non-conforming youth (persons under 18 years of age). This change allowed a number of additional analyses and offers important opportunities for future follow-up. STRONG stakeholders also helped to develop a comprehensive list of hormonal medications and procedures used for gender affirmation. The publications describing our formative research, ${ }^{2443-45}$ the current communication and the reports in preparation, all include stakeholders as coauthors.

\section{CONCLUSIONS}

Although the body of literature addressing transgender health issues has been growing, ${ }^{46}$ most studies focus on substance use, sexual health, sexually transmitted infections and, to a lesser extent, mental health conditions. ${ }^{47}$ By contrast, limited data are available on general health 
status, or the incidence of chronic age-related conditions including cardiovascular disease, endocrine disorders and cancer.

To date, most data on morbidity and mortality in transgender populations come from clinical centres in Europe. ${ }^{19-50}$ These studies are characterised by detailed clinical data; however, they are limited by relatively small sample sizes.

In terms of overall design and size, STRONG is comparable to US-based studies that used the Veterans Health Administration (VHA) data. ${ }^{22} 51$ Unlike our study, however, the VHA data did not distinguish between TF and TM subjects and were limited to transgender persons identified via ICD-9 codes, without keywords.

We recognise that transgender people enrolled through an integrated healthcare system will yield a cohort of persons with health insurance. Weighing against this concern is the demonstrated ability to cost-effectively identify a large cohort of transgender subjects and referents with high degree of internal validity. The availability of a well-defined underlying population with detailed EMR ensures that participation does not require subject opt-in and allows selecting a complete cohort (rather than a sample) of eligible subjects. Moreover, as KP now provides 'one-stop' delivery of transgender care, the likelihood of capturing full details of gender affirmation treatment is increased. These internal validity advantages weigh heavily as a counter against concerns about representativeness.

In summary, STRONG is well positioned to fill existing knowledge gaps and make important contributions to the current literature. Lessons learnt while conducting this project provide support to future transgender health-related research. The methodology can be implemented at other healthcare institutions with EMRs, particularly in organisations participating in the Healthcare Systems Research Network (total population of almost 20 million) with little site-specific customisation. With extended follow-up and expanded cohort size, the data will permit additional analyses of rare health endpoints across various categories of surgical procedures, and different HT formulations, routes of administration and doses.

\section{Author affiliations}

${ }^{1}$ Department of Research \& Evaluation, Kaiser Permanente Southern California, Pasadena, California, USA

${ }^{2}$ Department of Epidemiology, Rollins School of Public Health, Emory University, Atlanta, Georgia, USA

${ }^{3}$ Division of Research, Kaiser Permanente Northern California (emerita), Oakland, California, USA

${ }^{4}$ Center for Clinical and Outcomes Research, Kaiser Permanente Georgia, Atlanta, Georgia, USA

${ }^{5}$ The Rockway Institute, Alliant International University, San Francisco, California, USA

${ }^{6}$ Division of Research, Kaiser Permanente Northern California, Oakland, California, USA

${ }^{7}$ School of Public Health, Georgia State University, Atlanta, Georgia, USA

${ }^{8}$ The Permanente Medical Group, Kaiser Permanente Northern California, Oakland, California, USA

${ }^{9}$ Emory University School of Medicine, Atlanta, Georgia, USA

${ }^{10}$ The Atlanta VA Medical Center, Atlanta, Georgia, USA
Acknowledgements The authors acknowledge Kimberly L. Cannavale, MPH, and Alexander S Carruth who performed additional validations of eligibility and ascertainment of natal sex via reviews of medical records.

Contributors VPQ and MG prepared the original draft of the manuscript. RN conducted data analyses and put together tables and figures. RC and LC were responsible for the preparation and application of data collection programs and ascertainment of study variables. EH, VPQ, DG, MJS and DT led study implementation at participating sites and were actively involved in study planning and design. TAB-C, AM and BR were responsible for the day-to-day project management at each site. DR and TLL provided methodological input on various aspects of study design, including identification of sources of bias and ways of addressing threats to validity. VT and JS provided input on clinical aspects of the study and were responsible for adjudication of study eligibility, gender identity and gender affirmation. KW developed, pilot-tested and supervised the cohort validation and gender identity ascertainment review protocols and programming. SG, CV and SW are members of the Stakeholder Advisory Group who assisted with the development of inclusion and exclusion criteria, assessment of gender identity and interpretation of the data. All authors provided critical review of the manuscript for important intellectual content and approved the final version.

Funding This research was supported by the Contract AD-12-11-4532 from the Patient Centered Outcome Research Institute and by the Grant R21HD076387 from the Eunice Kennedy Shriver National Institute of Child Health and Human Development.

Competing interests None declared.

Ethics approval Emory University IRB.

Provenance and peer review Not commissioned; externally peer reviewed.

Data sharing statement Once the initial data analyses are complete, we will be open to collaborations with outside investigators as permitted by the IRBS of participating sites as well as by local, state and federal laws and regulations. In particular, we will encourage collaborations with researchers whose expertise is under-represented on our research team. To become a collaborator, a researcher will be required to submit an application, which will undergo both a scientific and an IRB review. In view of the complexity of the database, interested investigators will be asked to form a collaborative arrangement with the STRONG investigators rather than simply receive the data themselves. No additional data are available.

Open Access This is an Open Access article distributed in accordance with the Creative Commons Attribution Non Commercial (CC BY-NC 4.0) license, which permits others to distribute, remix, adapt, build upon this work non-commercially, and license their derivative works on different terms, provided the original work is properly cited and the use is non-commercial. See: http://creativecommons.org/ licenses/by-nc/4.0/

(c) Article author(s) (or their employer(s) unless otherwise stated in the text of the article) 2017. All rights reserved. No commercial use is permitted unless otherwise expressly granted.

\section{REFERENCES}

1. Safer JD, Coleman E, Hembree W. There is reason for optimism: an introduction to the special issue on research needs in transgender health and medicine. Curr Opin Endocrinol Diabetes Obes 2016;23:165-7.

2. Lombardi E. Enhancing transgender health care. Am J Public Health 2001;91:869-72.

3. Bockting W, Coleman E, De Cuypere G. Care of transsexual persons. N Engl J Med 2011;364:2559-60.

4. Bockting W. From construction to context: Gender through the eyes of the transgendered. Siecus Report 1999;28:3-7.

5. Giami A, Beaubatie E. Gender identification and sex reassignment surgery in the trans population: a survey study in France. Arch Sex Behav 2014;43:1491-501.

6. Reisner SL, Gamarel KE, Dunham E, et al. Female-to-male transmasculine adult health: a mixed-methods community-based needs assessment. J Am Psychiatr Nurses Assoc 2013;19:293-303.

7. Reisner SL, Radix A, Deutsch MB. Integrated and gender-affirming transgender clinical care and research. J Acquir Immune Defic Syndr 2016;72(Suppl 3):S235-42.

8. Knudson G, De Cuypere G, Bockting W. Recommendations for revision of the DSM diagnoses of gender identity disorders: consensus statement of the World Professional Association for Transgender Health. Int J Transgend 2010;12:115-8. 
9. Coleman E, Bockting W, Botzer M, et al. Standards of Care for the health of transsexual, transgender, and gender-nonconforming people, version 7. Int J Transgend 2012;13:165-232.

10. Hembree WC, Cohen-Kettenis P, Delemarre-van de Waal HA, et al. Endocrine treatment of transsexual persons: an Endocrine Society clinical practice guideline. J Clin Endocrinol Metab 2009;94:3132-54

11. Institute of Medicine. The health of lesbian, gay, bisexual, and transgender people: building a foundation for better understanding. Washington, DC: The National Academies Press, 2011.

12 The Women's Health Initiative Study Group. Design of the women's health initiative clinical trial and observational study. Control Clin Trials 1998;19:61-109.

13. Anderson GL, Limacher M, Assaf AR, et al. Effects of conjugated equine estrogen in postmenopausal women with hysterectomy: the Women's Health Initiative randomized controlled trial. JAMA 2004;291:1701-12.

14. Cattrall FR, Healy DL. Long-term metabolic, cardiovascular and neoplastic risks with polycystic ovary syndrome. Best Pract Res Clin Obstet Gynaecol 2004;18:803-12.

15. Schneider C, Cerwenka S, Nieder TO, et al. Measuring gender dysphoria: A multicenter examination and comparison of the Utrecht Gender Dysphoria Scale and the Gender Identity/Gender Dysphoria Questionnaire for adolescents and adults. Arch Sex Behav 2016;45:551-8.

16. Feldman J, Brown GR, Deutsch MB, et al. Priorities for transgender medical and healthcare research. Curr Opin Endocrinol Diabetes Obes 2016;23:180-7.

17. Reisner SL, Deutsch MB, Bhasin S, et al. Advancing methods for US transgender health research. Curr Opin Endocrinol Diabetes Obes 2016;23:198-207.

18. Dekker MJ, Wierckx K, Van Caenegem E, et al. A European Network for the Investigation of Gender Incongruence: endocrine part. J Sex Med 2016:13:994-9.

19. Kreukels BP, Haraldsen IR, De Cuypere G, et al. A European Network for the Investigation of Gender Incongruence: the ENIGI initiative. Eur Psychiatry 2012;27:445-50.

20. Reisner SL, Poteat T, Keatley J, et al. Global health burden and needs of transgender populations: a review. Lancet 2016;388:412-36.

21. Meier SC, Pardo ST, Labuski C, et al. Measures of clinical health among female-to-male transgender persons as a function of sexual orientation. Arch Sex Behav 2013;42:463-74.

22. Blosnich JR, Brown GR, Shipherd Phd JC, et al. Prevalence of gender identity disorder and suicide risk among transgender veterans utilizing Veterans Health Administration care. Am J Public Health 2013;103:e27-e32.

23. Brown GR, Jones KT. Racial health disparities in a cohort of 5,135 transgender veterans. J Racial Ethn Health Disparities 2014:1:257-66.

24. Roblin D, Barzilay J, Tolsma D, et al. A novel method for estimating transgender status using electronic medical records. Ann Epidemiol 2016;26:198-203.

25. Centers for Medicare \& Medicaid Services. Medicare and Medicaid programs; electronic health record incentive program-stage 3 and modifications to meaningful use in 2015 through 2017. Rockville, MD: Department of Health and Human Services, 2015.

26. Cahill S, Singal R, Grasso C, et al. Do ask, do tell: high levels of acceptability by patients of routine collection of sexual orientation and gender identity data in four diverse American community health centers. PLoS One 2014:9:e107104.

27. Deutsch MB. Gender-affirming surgeries in the era of insurance coverage: developing a framework for psychosocial support and care navigation in the perioperative period. $J$ Health Care Poor Underserved 2016;27:386-91.

28. Shipherd JC, Kauth MR, Matza A. Nationwide interdisciplinary e-consultation on transgender care in the Veterans Health Administration. Telemed J E Health 2016;22:1008-12.
29. Stevens J, Gomez-Lobo V, Pine-Twaddell E. Insurance coverage of puberty blocker therapies for transgender youth. Pediatrics 2015;136:1029-31.

30. Tangpricha V, den Heijer M. Oestrogen and anti-androgen therapy for transgender women. Lancet Diabetes Endocrinol 2017;5.

31. Quinn VP, Becerra TA, Gillespie T, et al. Embedding patients, providers, and community stakeholders in research to improve transgender health. J Patient Cent Res Rev 2015;2:114-5.

32. Gabriel SE, Normand SL. Getting the methods right-the foundation of patient-centered outcomes research. N Engl J Med 2012;367:787-90.

33. Koebnick C, Langer-Gould AM, Gould MK, et al. Sociodemographic characteristics of members of a large, integrated health care system: comparison with US Census Bureau data. Perm J 2012;16:37-41.

34. Gordon NP. How does the adult Kaiser Permanente membership in Northern California compare with the larger community? Oakland, CA: Kaiser Permanente Division of Research, 2006.

35. Lieu TA, Hinrichsen VL, Moreira A, et al. Collaborations in populationbased health research: the 17th annual HMO Research Network conference, March 23-25, 2011, Boston, Massachusetts, USA. Clin Med Res 2011:9:137-40.

36. Simon GE, Stewart C, Beck A, et al. National prevalence of receipt of antidepressant prescriptions by persons without a psychiatric diagnosis. Psychiatr Serv 2014;65:944-6.

37. Go AS, Magid DJ, Wells B, et al. The Cardiovascular Research Network: a new paradigm for cardiovascular quality and outcomes research. Circ Cardiovasc Qual Outcomes 2008;1:138-47.

38. Goodman M, Fletcher RH, Doria-Rose VP, et al. Observational methods to assess the effectiveness of screening colonoscopy in reducing right colon cancer mortality risk: SCOLAR. J Comp Eff Res 2015;4:541-51.

39. Brown GR, Jones KT. Incidence of breast cancer in a cohort of 5,135 transgender veterans. Breast Cancer Res Treat 2015;149:191-8.

40. World Health Organisation. International Statistical Classification of Diseases and Related Health Problems. 9th Revision. Geneva: WHO. 1978.

41. Maguire N. In search of the "V" codes (ICD-9-CM). J Med Pract Manage 2012;28:56-7.

42. Hughes IA. Disorders of sex development: a new definition and classification. Best Pract Res Clin Endocrinol Metab 2008;22:119-34.

43. Owen-Smith A, Sineath RC, Sanchez T, et al. Perception of community tolerance and prevalence of depression among transgender persons. J Gay Lesbian Ment Healt 2017;21:64-76.

44. Owen-Smith AA, Woodyatt C, Sineath RC, et al. Perceptions of barriers to and facilitators of participation in health research among transgender people. Transgend Health 2016;1:187-96.

45. Sineath RC, Woodyatt C, Sanchez T, et al. Determinants of and barriers to hormonal and surgical treatment receipt among transgender people. Transgend Health 2016;1:129-36.

46. Collin L, Tangpricha V, Goodman M. Worldwide prevalence of transgender and gender non-conformity. In: Ettner R, Monstrey S, Coleman E, eds. Principles of transgender medicine and surgery. New York, NY: Routledge, 2016.

47. MacCarthy S, Reisner SL, Nunn A, et al. The time is now: attention increases to transgender health in the United States but scientific knowledge gaps remain. LGBT Health 2015;2:287-91.

48. van Kesteren PJ, Asscheman H, Megens JA, et al. Mortality and morbidity in transsexual subjects treated with cross-sex hormones. Clin Endocrinol 1997;47:337-43.

49. Asscheman H, Giltay EJ, Megens JA, et al. A long-term follow-up study of mortality in transsexuals receiving treatment with cross-sex hormones. Eur J Endocrinol 2011;164:635-42.

50. Dhejne C, Lichtenstein P, Boman M, et al. Long-term follow-up of transsexual persons undergoing sex reassignment surgery: cohort study in Sweden. PLoS One 2011;6:e16885.

51. Kauth MR, Shipherd JC, Lindsay J, et al. Access to care for transgender veterans in the Veterans Health Administration: 20062013. Am J Public Health 2014;104(Suppl 4):S532-4. 